\title{
The pancreatic exocrine function in patients with pancreatic endocrine insufficiency: the evaluation with cine-dynamic magnetic resonance cholangiopancreatography using a spatially selective inversion-recovery pulse and T1 mapping
}

\author{
Mayumi Higashi $^{1}$ D $\cdot$ Masahiro Tanabe $^{1} \cdot$ Teppei Yonezawa $^{1} \cdot$ Matakazu Furukawa $^{1} \cdot$ Etsushi lida $^{1} \cdot \mathrm{Katsuyoshi}$ Ito $^{1}$
}

Received: 11 December 2021 / Accepted: 16 February 2022 / Published online: 2 March 2022

(c) The Author(s) 2022

\begin{abstract}
Purpose To evaluate the association of the pancreatic exocrine function estimated by cine-dynamic magnetic resonance cholangiopancreatography (MRCP) using a spatially selective inversion-recovery (IR) pulse with the pancreatic endocrine function estimated by the $\mathrm{T} 1$ relaxation time of the pancreatic parenchyma and $\mathrm{HbAlc}$ values.

Materials and methods Forty-three patients with suspected hepatobiliary or pancreatic diseases were included. Patients were classified into three groups: HbA1c $<5.7 \%$ (normal group), $5.7 \% \leq \mathrm{HbA} 1 \mathrm{c}<6.5 \%$ (prediabetes group), and $\mathrm{HbA} 1 \mathrm{c} \geq 6.5 \%$ (diabetes group). The frequency of the secretory flow of the pancreatic juice was observed within the area of the IR pulse, and the moving distance (mean secretion grade) of the pancreatic juice inflow within the area of the IR pulse on cinedynamic MRCP, and the T1 relaxation time of the pancreatic parenchyma on the T1 map images were assessed. The MR imaging measurements were compared using Spearman's rank correlation coefficient analysis and the Kruskal-Wallis and Mann-Whitney $U$ tests.

Results Both the mean secretion grade and frequency of the pancreatic secretory inflow had a significant negative correlation with the T1 relaxation time of the pancreatic parenchyma ( $r=-0.335, p=0.028$ and $r=-0.305, p=0.047$, respectively) and HbA1c values ( $r=-0.308, p=0.044$ and $r=-0.313, p=0.041$, respectively). Both the mean secretion grade and frequency of the pancreatic secretory inflow in the elevated $\mathrm{HbA1c}$ (prediabetes and diabetes) group were significantly lower than those in the normal group ( $p=0.030$ and $p=0.029$, respectively).

Conclusion The pancreatic exocrine function estimated by cine-dynamic MRCP was significantly lower in patients with prediabetes and diabetes than in controls. Cine-dynamic MRCP with a spatially selective IR pulse may be useful for the early diagnosis of pancreatic exocrine insufficiency in patients with pancreatic endocrine insufficiency.
\end{abstract}

Keywords Pancreas $\cdot$ Cine-dynamic MRCP $\cdot$ MR imaging $\cdot$ Diabetes mellitus

\section{Introduction}

The endocrine and exocrine tissues of the pancreas reciprocally interact to coordinate the activities of digestion, absorption, and glucose metabolisms [1]. Insulin, as the islet-derived hormone, regulates the exocrine function and mediates the interplay between the endocrine and exocrine

Mayumi Higashi

mhigashi@yamaguchi-u.ac.jp

1 Department of Radiology, Yamaguchi University Graduate School of Medicine, 1-1-1 Minami-Kogushi, Ube, Yamaguchi 755-8505, Japan pancreas [2]. Therefore, impairment of the pancreatic endocrine function can cause pancreatic exocrine insufficiency.

Diabetes mellitus (DM) is one of the most common endocrine disorders, resulting from insulin resistance and insulin deficiency and accompanied by an impaired exocrine function that manifests as an insufficient activity of digestive enzymes. Pancreatic exocrine insufficiency has been reported to be present in a considerable proportion of patients with DM (26\%-74\% of type $1 \mathrm{DM}$ patients and $10 \%-56 \%$ of type $2 \mathrm{DM}$ patients) [3]. Patients with pancreatic exocrine insufficiency often have symptoms including steatorrhea, abdominal pain, abdominal bloating and weight loss, mainly due to the maldigestion and malabsorption of fat 
[4], and these symptoms can significantly affect the quality of life. Therefore, the early and accurate diagnosis of pancreatic exocrine insufficiency is essential for the effective treatment and improvement of the quality of life in DM patients.

To facilitate the early diagnosis of pancreatic exocrine insufficiency, indirect pancreatic function tests, especially the measurement of fecal elastase-1 (FE-1) in spot stool, have been used in clinical practice. FE-1 has demonstrated good sensitivity and specificity in moderate and severe pancreatic exocrine insufficiency (sensitivity, 100\%; specificity, 93\%); however, in mild cases of pancreatic exocrine insufficiency, the sensitivity is inadequate (sensitivity, 63\%) [5].

Magnetic resonance imaging (MRI) have been increasingly used to evaluate the impairment of the pancreatic endocrine and exocrine function in patients with pancreatic diseases, such as DM and chronic pancreatitis (CP) [6-11]. A previous study demonstrated that cine-dynamic MR cholangiopancreatography (MRCP) with a spatially selective inversion-recovery (IR) pulse capable of visualizing the secretory flow of pancreatic juice had potential utility for estimating the pancreatic exocrine function noninvasively [6]. Another study showed that the T1 relaxation time of the pancreatic parenchyma measured by $\mathrm{T} 1$ mapping, which may reflect parenchymal fibrotic changes, was significantly increased in patients with an impaired glucose tolerance [11]. However, no previous study has evaluated the pancreatic exocrine function in patients with impaired glucose tolerance and DM using MRI.

Therefore, the present study evaluated the association of the pancreatic exocrine function estimated by cine-dynamic MRCP using a spatially selective IR pulse with the pancreatic endocrine function estimated by the $\mathrm{T} 1$ relaxation time of the pancreatic parenchyma and $\mathrm{HbAlc}$ values.

\section{Materials and methods}

\section{Study population}

The institutional review board of our hospital approved this retrospective study, and the need for patient informed consent was waived. Using our radiology reporting database system, we searched for patients suspected of having hepatobiliary or pancreatic diseases who underwent abdominal MRI, including T1 mapping and cine-dynamic MRCP with a spatially selective IR pulse, between March 2019 and January 2020.

Among these patients, we identified 49 patients who underwent an $\mathrm{HbA} 1 \mathrm{c}$ test within 1 month before or after MRI because of the clinical requirement for the assessment of glucose tolerance. Six of these patients were excluded for the following reasons: incomplete cine-dynamic MRCP $(n=1)$; unclear visualization of the main pancreatic duct $(n=2)$; large tumors of the pancreatic head that hampered the evaluation of the pancreatic juice inflow on cinedynamic MRCP $(n=2)$; and severe fatty infiltration $(n=1)$. Thus, 43 consecutive patients ( 26 men, 17 women; mean age, $70.4 \pm 11.3$ years; range, $26-88$ years) were included in our study. These included patients with pancreatic diseases $(n=19)$, such as branch-duct intraductal papillary mucinous neoplasms ( $n=12$, median size $8.5 \mathrm{~mm}$, interquartile range, $7.75-12.75 \mathrm{~mm})$, pancreatic cyst $(n=1)$, pancreatic ductal dilatation with unknown causes $(n=4)$ and chronic pancreatitis $(n=2)$; patients with hepatobiliary diseases $(n=22)$ such as acute cholecystitis $(n=4)$, biliary stones $(n=5)$, cholangitis $(n=3)$, adenomyomatosis $(n=2)$, choledochal cyst $(n=1)$, peribiliary cyst $(n=1)$, intrahepatic bile ductal dilatation with unknown cause $(n=1)$, gallbladder polyp $(n=1)$, gallbladder cancer $(n=1)$, hepatocellular carcinoma $(n=1)$, hepatic hemangioma $(n=1)$ and fatty liver $(n=1)$; and patients with no diseases $(n=2)$. The patients were classified into three groups based on the American Diabetes Association criteria using HbA1c values as follows: normal group, $\mathrm{HbA1c}<5.7 \%(n=12)$; prediabetes group, $5.7 \% \leq \mathrm{HbA} 1 \mathrm{c}<6.5 \%(n=15)$; and diabetes group, $\mathrm{HbA} 1 \mathrm{c} \geq 6.5 \%(n=16)$.

\section{MRI technique}

MRI was performed with a 3.0-T MR system (Vantage Galan ZGO; Canon Medical Systems, Tochigi, Japan) equipped with a 16-channel body coil. Patients were required to fast for at least $4 \mathrm{~h}$ before MR examinations. At the MR examination, $36 \mathrm{mg}$ of manganese chloride tetrahydrate (Bothdel Oral Solution, 250 mL; Kyowa Hakko Kirin, Tokyo, Japan) was ingested orally to reduce the signal from the bowel. Cine-dynamic MRCP with a spatially selective IR pulse and T1 mapping were routinely performed as part of our hepatobiliary and pancreatic MR examination protocol. First, a breath-hold, thick-slab 2D MRCP was obtained as a reference image to depict the main pancreatic duct in the coronal plane. The imaging parameters were as follows: repetition time (TR)/echo time (TE), 5000/507 ms; echo train spacing, $6.5 \mathrm{~ms}$; slice thickness, $50 \mathrm{~mm}$; acquisition matrix, $512 \times 512$; field of view (FOV), $350 \times 350 \mathrm{~mm}$; and bandwidth, $558 \mathrm{~Hz} /$ pixel. Then, using the same MR sequence, a spatially selective IR pulse (inversion time, $2200 \mathrm{~ms}$ ) with a width of $20 \mathrm{~mm}$ was placed on the pancreatic head perpendicular to the main pancreatic duct to null the static pancreatic juice signal. With this method, the static pancreatic juice in the areas subjected to a spatially selective IR pulse appeared dark, while the inflow of the pancreatic juice in these areas was observed as a high signal intensity. MRCP with a spatially selective IR pulse was repeatedly performed every $15 \mathrm{~s}(5 \mathrm{~s}$ of scanning and $10 \mathrm{~s}$ of rest) during $5 \mathrm{~min}$ (total of 20 images), and a series of these MRCP images was 
defined as cine-dynamic MRCP with a spatially selective IR pulse (Fig. 1).

T1 maps were acquired using the modified Look-Locker method. The following imaging parameters were used: TR/TE, 3.4/1.3 ms; flip angle, $13^{\circ}$; slice number, 1 slice; slice thickness, $8 \mathrm{~mm}$; acquisition matrix, $192 \times 256$; FOV, $280 \times 360 \mathrm{~mm}$; parallel imaging factor, 2; and band width, $781 \mathrm{~Hz} /$ pixel.

\section{Image analyses}

Cine-dynamic MRCP images were evaluated independently by 2 radiologists (M.H. and K.I., with 5 and 32 years of clinical experience, respectively) who were blinded to any clinical information of the patients, and any interpretation discrepancies were resolved by reaching a consensus. MR images were evaluated for (a) the frequency of the pancreatic juice secretion, defined as the frequency of the detection of a high signal inflow of the pancreatic juice within the area with a spatially selective IR pulse, and (b) the secretion grade of the pancreatic juice, which was assigned according to the distance that the pancreatic juice flowed into the area of a spatially selective IR pulse using the 5-point secretion grading score (grade 0 , no secretion; grade $1,<5 \mathrm{~mm}$; grade 2, 5-10 mm; grade 3, 11-15 mm; grade 4,> $15 \mathrm{~mm}$ ) (Fig. 2). The mean secretion grade was defined as follows:

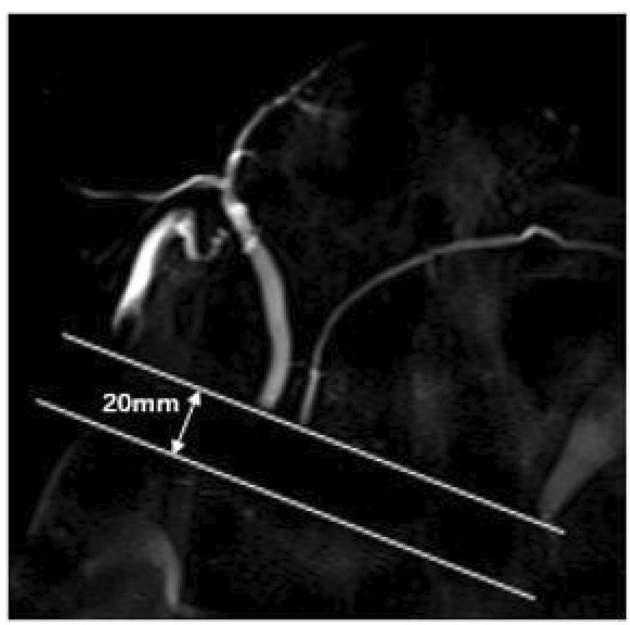

a

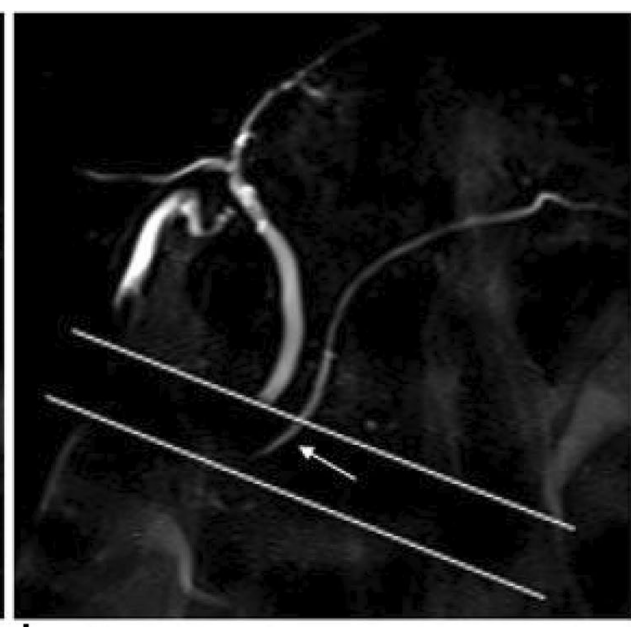

b
Fig. 1 Imaging findings of cine dynamic MRCP with a spatially selective IR pulse. a A spatially selective IR pulse (inversion time, $2200 \mathrm{~ms}$ ) with a width of $20 \mathrm{~mm}$ was placed on the pancreatic head perpendicular to the main pancreatic duct to null the static pancreatic juice signal. The static pancreatic juice within the area of the spatially selective IR pulse was shown as low signal intensity. b The secretory flow of the pancreatic juice was observed as high signal inten- sity (arrow) within the area of the spatially selective IR pulse. The secretion grade of the pancreatic juice was categorized as grade 3 . MRCP with a spatially selective IR pulse was repeatedly performed every $15 \mathrm{~s}$ ( $5 \mathrm{~s}$ of scanning and $10 \mathrm{~s}$ of rest) during 5 min (total of 20 images), and a series of these MRCP images was defined as cinedynamic MRCP with a spatially selective IR pulse
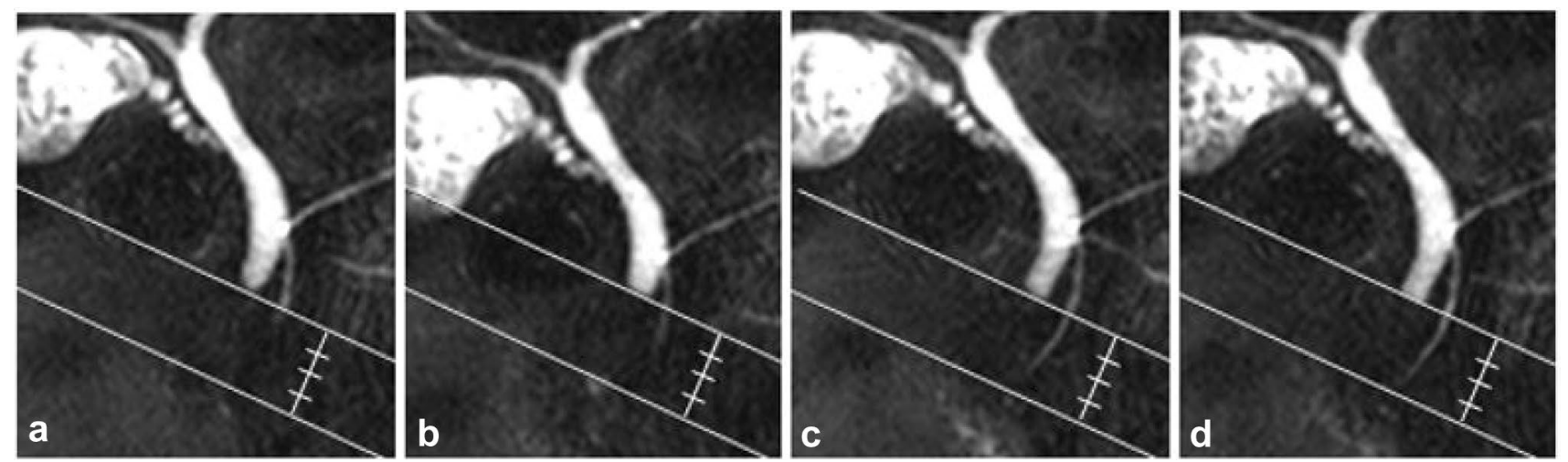

Fig. 2 Categorization of the secretion grade based on the distance that the pancreatic juice flowed within the area of the spatially selective IR pulse. a Grade 1 (less than $5 \mathrm{~mm})$, b grade $2(5-10 \mathrm{~mm})$, c grade $3(11-15 \mathrm{~mm})$, d grade 4 (more than $15 \mathrm{~mm}$ ) 
(total grading score)/20. In addition, the reviewers measured the $\mathrm{T} 1$ relaxation time of the pancreatic parenchyma on the T1 map images using operator-defined regions of interest (ROIs), in consensus. Effort was made to draw the ROI circles as large as possible in a homogeneous area of the pancreatic parenchyma while avoiding the dilated pancreatic duct, retroperitoneal fat and artifacts. The ROI measurements were made at two places on the body or tail of the pancreas, and the average values were used for the analysis (Fig. 3).

\section{Statistical analyses}

Statistical analyses were performed using the SPSS software program for Windows, version 18.0 (IBM Corp., Armonk, NY, USA). A Spearman's rank correlation coefficient analysis was performed to evaluate the correlation between the MRI measurements and HbA1c values. Kruskal-Wallis and Mann-Whitney $U$ tests were conducted to compare the MRI measurements among the groups. A $p$ value less than 0.05 was considered to indicate a significant difference. The interobserver agreement between the two radiologists was also evaluated using Cohen $\kappa$ statistics. We interpreted the $\kappa$ values as follows: $\leq 0.40$, poor agreement; $0.41-0.60$, moderate agreement; $0.61-0.80$, substantial agreement; and $\geq 0.81$, excellent agreement.

\section{Results}

In the $\mathrm{HbA} 1 \mathrm{c}$ test, the mean $\mathrm{HbA} 1 \mathrm{c}$ value was $6.3 \% \pm 0.9 \%$ (range 4.7\%-8.2\%) in all patients. The median T1 relaxation times of the pancreatic parenchyma on the T1 map

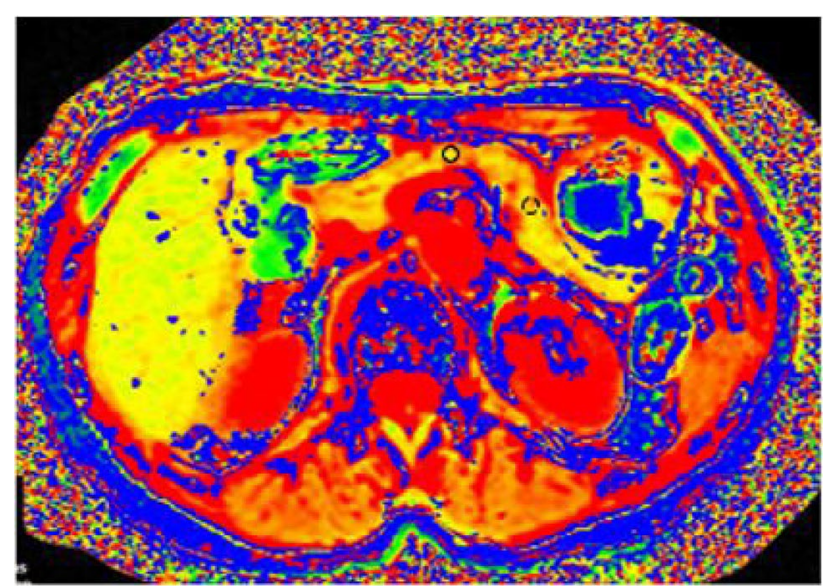

Fig. 3 T1 map image in an 81-year-old woman with prediabetes. The T1 relaxation times of body (circle ROI) and tail (dashed circle ROI) of the pancreas were $889 \mathrm{~ms}$ and $978 \mathrm{~ms}$, respectively. The mean T1 relaxation time was $934 \mathrm{~ms}$ images was $899 \mathrm{~ms}$ (range 656-1498 ms; interquartile range 811-1033 ms). In cine-dynamic MRCP with a spatially selective IR pulse, the median frequency of the pancreatic juice inflow in all patients was 7 times (range 0-20 times; interquartile range $1-14.5$ times) in a series of 20 images. The median mean secretion grade of the pancreatic juice in all patients was 0.35 (range $0-2.6$; interquartile range 0.075-0.9). The interobserver agreement for the detection of the inflow of the pancreatic juice and the grading of the moving distance of the pancreatic juice with cine-dynamic MRCP was excellent $(\kappa$ value $=0.927)$. Regarding the relationship of the HbA1c values with the mean secretion grade and frequency of the pancreatic secretory inflow, the HbA1c values had significant negative correlations with both the mean secretion grade $(r=-0.308, p=0.044)$ (Fig. 4) and the frequency of the pancreatic secretory inflow $(r=-0.313$, $p=0.041)$. In contrast, the $\mathrm{HbA} 1 \mathrm{c}$ values had a significant positive correlation with the T1 relaxation time of the pancreatic parenchyma $(r=0.408, p=0.007)$. In addition, the T1 relaxation time of the pancreatic parenchyma had significant negative correlations with both the mean secretion grade $(r=-0.335, p=0.028)$ (Fig. 5) and the frequency of the pancreatic secretory inflow $(r=-0.305, p=0.047)$.

In the comparison among the 3 groups (Table 1), the $\mathrm{T} 1$ relaxation time of the pancreatic parenchyma in the normal group (median, 803 [interquartile range 750-910] msec) was significantly shorter than that in the prediabetes (median, 934 [interquartile range 850-1024] msec) and diabetes groups (median, 968 [interquartile range 851-1191] msec) $(p=0.034)$. In cine-dynamic MRCP findings, no significant differences were noted in the mean secretion grade $(p=0.095)$ and frequency of the pancreatic secretory inflow ( $p=0.093$ ) among the 3 groups. However, there were trends toward significances in both the mean secretion grade (median, 0.8 [interquartile range $0.34-1.25$ ] vs. median, 0.1 [interquartile range $0.025-0.775$ ],$p=0.066$ ) and the

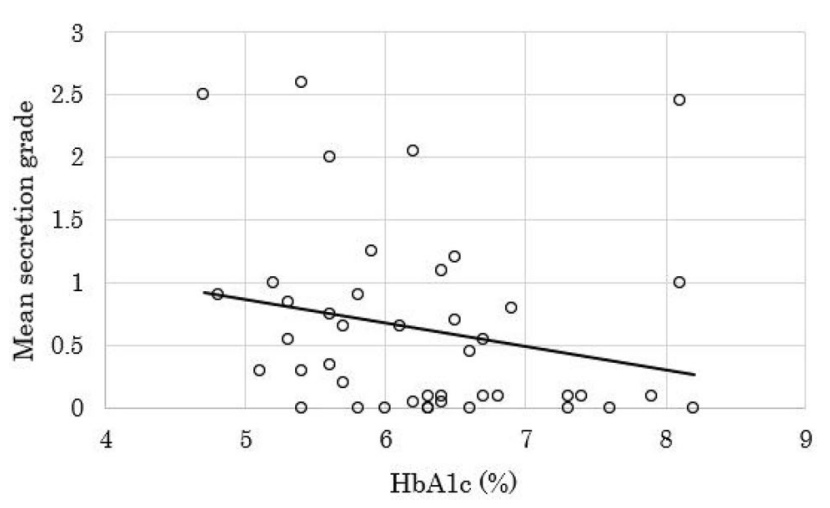

Fig. 4 Correlation between the mean secretion grade and HbA1c value. There was a significant negative correlation between the mean secretion grade and HbA1c value $(r=-0.308, p=0.044)$ 


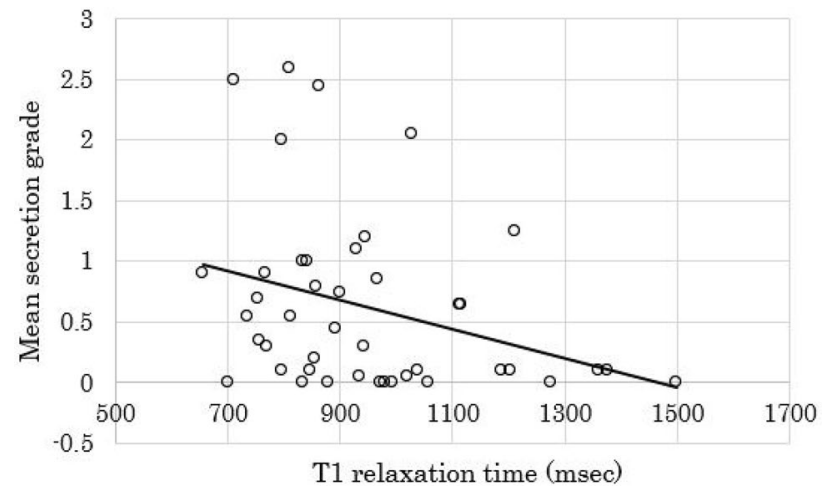

Fig. 5 Correlation between the mean secretion grade and T1 relaxation time of the pancreatic parenchyma. There was a significant negative correlation between the mean secretion grade and T1 relaxation time of the pancreatic parenchyma $(r=-0.335, p=0.028)$

frequency of the pancreatic secretory inflow (median, 12 [interquartile range 6.75-16] times vs. median, 2 [interquartile range $0.5-13.5$ ] times, $p=0.059$ ) between the normal group and the prediabetes group although the differences were not statistically significant. Comparing the prediabetes group and the diabetes group, the differences in $\mathrm{T} 1$ relaxation time, mean secretion grade and frequency of the pancreatic secretory inflow were not significant $(p=0.406$, $p=0.904$ and, $p=1.000$, respectively).

In the comparison between the normal group and the group with elevated $\mathrm{HbA1c}$ (i.e. prediabetes and diabetes, $n=31$ ) (Table 2), the frequency of the pancreatic secretory inflow was significantly lower in the elevated $\mathrm{HbAlc}$ group than in the normal group (median, 2 [interquartile range $0.5-12$ ] times vs. median, 12 [interquartile range 6.75-16] times, $p=0.029$ ). In addition, the mean secretion grade was also significantly lower in the elevated $\mathrm{HbA} 1 \mathrm{c}$ group than in the normal group (median, 0.1 [interquartile range $0.025-0.75$ ] vs. median, 0.8 [interquartile range $0.34-1.25$ ], $p=0.030$ ), indicating that the pancreatic juice secretion was significantly decreased in the patients with elevated HbA1c.

\section{Discussion}

Our findings showed that both the mean secretion grade and frequency of the pancreatic secretory inflow in cine-dynamic MRCP with a spatially selective IR pulse had a significant negative correlation with the HbA1c values. In addition, both the mean secretion grade and frequency of the pancreatic secretory inflow in the elevated $\mathrm{HbA} 1 \mathrm{c}$ group were significantly lower than those in the normal group. A previous study demonstrated that the cut-off value of 0.7 for the mean secretion grade in cine-dynamic MRCP was the criterion of pancreatic exocrine dysfunction [6]. This indicates that the patients in the elevated $\mathrm{HbA1c}$ group whose median mean secretion grade was 0.1 had an impaired pancreatic exocrine function. Therefore, our findings suggest that the pancreatic exocrine function might be reduced in patients with an impaired pancreatic endocrine function.

In this study, the $\mathrm{T} 1$ relaxation time of the pancreatic parenchyma showed a significant positive correlation with the HbAlc values, in accordance with a previous study [11]. In addition, we also found that both the mean secretion grade and frequency of the pancreatic secretory inflow showed a significant negative correlation with the T1 relaxation time of the pancreatic parenchyma. These findings suggested that the reduced pancreatic exocrine function was interrelated with the decline in the pancreatic endocrine function, and indicated that a combination of cine-dynamic MRCP with a spatially selective IR pulse and T1 mapping might have

Table 2 Comparison of the MR measurements between the normal $\mathrm{HbA1c}$ group and the elevated $\mathrm{HbA1c}$ group

\begin{tabular}{llll}
\hline Parameter & $\begin{array}{l}\text { Normal group } \\
(n=12)\end{array}$ & $\begin{array}{l}\text { Elevated HbA1c } \\
\text { group } \\
(n=31)\end{array}$ & $P$ \\
\hline $\begin{array}{l}\text { T1 relaxation time of } \\
\text { pancreas (msec) }\end{array}$ & $803(750-910)$ & $944(850-1085)$ & 0.013 \\
$\begin{array}{l}\text { Frequency of pancre- } \\
\text { atic juice secretion } \\
\text { observed }\end{array}$ & $12(6.75-16)$ & $2(0.5-12)$ & 0.029 \\
$\begin{array}{l}\text { Mean secretion grade } \\
\text { Mean }\end{array}$ & $0.8(0.34-1.25)$ & $0.1(0.025-0.75)$ & 0.030 \\
\hline
\end{tabular}

Data are median with 25 th and 75 th percentiles in parentheses
Table 1 Comparison of the MR measurements among the three groups

\begin{tabular}{lllll}
\hline Parameter & $\begin{array}{l}\text { Normal group } \\
(n=12)\end{array}$ & $\begin{array}{l}\text { Prediabetes group } \\
(n=15)\end{array}$ & $\begin{array}{l}\text { Diabetes group } \\
(n=16)\end{array}$ & $P$ \\
\hline $\begin{array}{c}\text { T1 relaxation time of } \\
\text { pancreas (msec) }\end{array}$ & $803(750-910)$ & $934(850-1024)$ & $968(851-1191)$ & 0.034 \\
$\begin{array}{c}\text { Frequency of pancreatic } \\
\text { juice secretion observed } \\
\text { Mean secretion grade }\end{array}$ & $12(6.75-16)$ & $2(0.5-13.5)$ & $2(0.75-9.5)$ & 0.093 \\
\hline
\end{tabular}

Data are median with 25 th and 75 th percentiles in parentheses 
potential for estimating both the pancreatic endocrine and exocrine function noninvasively cooperating with physiological pancreatic function tests.

In this study, both the mean secretion grade and frequency of the pancreatic secretory inflow tended to be lower in the patients with prediabetes and DM than in those with a normal pancreatic endocrine function. This suggests that the pancreatic exocrine function might already be decreased in patients with prediabetes. Previous studies on the pancreas histology of diabetic patients showed many histopathological findings and structural changes, such as atrophy with decreased acinar and beta-cell, chronic inflammation, fibrosis due to microvascular complications, arteriosclerosis, nerve fiber loss and morphological changes in the pancreatic duct [12-14], and these changes can contribute to the development of pancreatic exocrine insufficiency. Thus far, little has been reported concerning the pancreatic exocrine function in patients with prediabetes. However, a decreased pancreatic beta-cell mass, microvascular damage and neuropathy have been also reported in patients with prediabetes $[15$, 16]. Therefore, pancreatic exocrine insufficiency can be seen in patients with prediabetes and early DM who have mild pancreatic endocrine dysfunction. In addition, our results suggest that cine-dynamic MRCP with a spatially selective IR pulse may be useful in the early diagnosis of pancreatic exocrine dysfunction in patients with mild pancreatic endocrine insufficiency, so early therapeutic intervention for pancreatic exocrine dysfunction is clinically desirable for patients with prediabetes or DM.

While we expected that the pancreatic exocrine function would be reduced with the progression of pancreatic endocrine dysfunction, no significant differences in the mean secretion grade or frequency of the pancreatic secretory inflow were observed between the patients with prediabetes and those with DM. In a cohort study of 667 patients with DM, Larger et al. [17] found no correlation between pancreatic exocrine insufficiency and the duration of DM. A long-term follow-up study suggested that mild to moderate pancreatic exocrine insufficiency had been present since the onset of DM and had not progressed [18]. These data suggest that the pancreatic exocrine function might not be significantly decreased during the transition from prediabetes to diabetes.

Several limitations associated with the present study warrant mention. First, because this study was retrospective and the number of patients was relatively small, there may have been potential selection bias. Second, the evaluation of the pancreatic secretory inflow within the area with a spatially selective IR pulse in cine-dynamic MRCP images may be subjective. However, the interobserver agreement for the assessment of the inflow of the pancreatic juice with cine-dynamic MRCP was excellent. Third, study population included patients with branch-duct intraductal papillary mucinous neoplasms which could increase the viscosity of pancreatic juice. However, the size of IPMNs was relatively small, and no patients showed the dilated pancreatic duct suggesting stagnant pancreatic flow. Therefore, it is less likely to affect the flow dynamics of pancreatic juice in these patients with IPMNs, and we believe that the validity of this study will not be compromised. Finally, we classified patients into three groups according to the $\mathrm{HbA} 1 \mathrm{c}$ values. Although the measurement of $\mathrm{HbA} 1 \mathrm{c}$ is adequate for the diagnosis of diabetes as well as the measurement of fasting plasma glucose and 2-h plasma glucose value during a 75-g oral glucose tolerance test, HbA1c is an indirectly measured value of the average blood glucose levels and may be influenced by a number of factors, such as age, race and presence of anemia [19]. Therefore, further clinical studies using other pancreatic endocrine function tests, including the measurement of C-peptide and glucagon levels, will be needed to validate our results.

In conclusion, the pancreatic exocrine function estimated by cine-dynamic MRCP with a spatially selective IR pulse was significantly decreased in patients with prediabetes and $\mathrm{DM}$, suggesting the interrelation between the pancreatic exocrine and endocrine functions. Cine-dynamic MRCP with a spatially selective IR pulse may contribute to the diagnosis of pancreatic exocrine insufficiency in patients with pancreatic endocrine insufficiency.

\section{Declarations}

Conflict of interest No financial support for this study was provided, and we have no disclosure.

Ethical approval This study was approved by the institutional review board.

Open Access This article is licensed under a Creative Commons Attribution 4.0 International License, which permits use, sharing, adaptation, distribution and reproduction in any medium or format, as long as you give appropriate credit to the original author(s) and the source, provide a link to the Creative Commons licence, and indicate if changes were made. The images or other third party material in this article are included in the article's Creative Commons licence, unless indicated otherwise in a credit line to the material. If material is not included in the article's Creative Commons licence and your intended use is not permitted by statutory regulation or exceeds the permitted use, you will need to obtain permission directly from the copyright holder. To view a copy of this licence, visit http://creativecommons.org/licenses/by/4.0/.

\section{References}

1. Piciucchi M, Capurso G, Archibugi L, Delle Fave MM, Capasso M, Delle FG. Exocrine pancreatic insufficiency in diabetic patients: prevalence, mechanisms, and treatment. Int J Endocrinol. 2015;2015:595649. 
2. Saisho Y. Pancreas volume and fat deposition in diabetes and normal physiology: consideration of the interplay between endocrine and exocrine pancreas. Rev Diabet Stud: RDS. 2016;13:132-47.

3. Zsori G, Illes D, Terzin V, Ivany E, Czako L. Exocrine pancreatic insufficiency in type 1 and type 2 diabetes mellitus: do we need to treat it? A systematic review. Pancreatology. 2018;18:559-65.

4. Lindkvist B. Diagnosis and treatment of pancreatic exocrine insufficiency. World J Gastroenterol. 2013;19:7258-66.

5. Löser C, Möllgaard A, Fölsch UR. Faecal elastase 1: a novel, highly sensitive, and specific tubeless pancreatic function test. Gut. 1996;39:580-6.

6. Yasokawa K, Ito K, Tamada T, Yamamoto A, Hayashida M, Tanimoto D, et al. Noninvasive investigation of exocrine pancreatic function: feasibility of cine dynamic MRCP with a spatially selective inversion-recovery pulse. J Magn Reson Imaging JMRI. 2015;42:1266-71.

7. Yasokawa K, Ito K, Kanki A, Yamamoto A, Torigoe T, Sato T, et al. Evaluation of pancreatic exocrine insufficiency by cinedynamic MRCP using spatially selective inversion-recovery (IR) pulse: correlation with severity of chronic pancreatitis based on morphological changes of pancreatic duct. Magn Reson Imaging. 2018;48:70-3.

8. Tirkes T, Lin C, Fogel EL, Sherman SS, Wang Q, Sandrasegaran K. T1 mapping for diagnosis of mild chronic pancreatitis. J Magn Reson Imaging JMRI. 2017;45:1171-6.

9. Wang M, Gao F, Wang X, Liu Y, Ji R, Cang L, et al. Magnetic resonance elastography and $\mathrm{T} 1$ mapping for early diagnosis and classification of chronic pancreatitis. J Magn Reson Imaging JMRI. 2018;48:837-45.

10. Noda Y, Goshima S, Tanaka K, Osada S, Tomita H, Hara A, et al. Findings in pancreatic MRI associated with pancreatic fibrosis and HbA1c values. J Magn Reson Imaging JMRI. 2016;43:680-7.

11. Noda Y, Goshima S, Tsuji Y, Kajita K, Kawada H, Kawai N, et al. Correlation of quantitative pancreatic $\mathrm{T} 1$ value and $\mathrm{HbA} 1 \mathrm{c}$ value in subjects with normal and impaired glucose tolerance. J Magn Reson Imaging JMRI. 2019;49:711-8.

12. Mohapatra S, Majumder S, Smyrk TC, Zhang L, Matveyenko A, Kudva YC, et al. Diabetes mellitus is associated with an exocrine pancreatopathy: conclusions from a review of literature. Pancreas. 2016;45:1104-10.

13. Hardt PD, Ewald N. Exocrine pancreatic insufficiency in diabetes mellitus: a complication of diabetic neuropathy or a different type of diabetes? Exp Diabetes Res. 2011;2011:761950.

14. Yagihashi S, Mizukami H, Sugimoto K. Mechanism of diabetic neuropathy: where are we now and where to go? J Diabetes Investig. 2011;2:18-32.

15. Yoneda S, Uno S, Iwahashi H, Fujita Y, Yoshikawa A, Kozawa J, et al. Predominance of beta-cell neogenesis rather than replication in humans with an impaired glucose tolerance and newly diagnosed diabetes. J Clin Endocrinol Metab. 2013;98:2053-61.

16. Singleton JR, Smith AG, Russell JW, Feldman EL. Microvascular complications of impaired glucose tolerance. Diabetes. 2003;52:2867-73.

17. Larger E, Philippe MF, Barbot-Trystram L, Radu A, Rotariu M, Nobécourt E, et al. Pancreatic exocrine function in patients with diabetes. Diabet Med: J Br Diabet Assoc. 2012;29:1047-54.

18. Creutzfeldt W, Gleichmann D, Otto J, Stockmann F, Maisonneuve P, Lankisch PG. Follow-up of exocrine pancreatic function in type-1 diabetes mellitus. Digestion. 2005;72:71-5.

19. American Diabetes A. 2. Classification and diagnosis of diabetes: standards of medical care in diabetes-2018. Diabetes Care. 2018;41:13-27.

Publisher's Note Springer Nature remains neutral with regard to jurisdictional claims in published maps and institutional affiliations. 